\title{
Does the Number of ICSI Cycles Performed per day or the Number of Oocytes Injected per day has an Impact on the Cycles' Outcome?
}

\author{
Amanda S. Setti ${ }^{1,2,3}$, Daniela P.A.F. Braga ${ }^{1,2}$, Rita Figueiraa ${ }^{1,3}$, Assumpto Iaconelli Jr. ${ }^{1,2}$, Tsutomu Aoki ${ }^{3}$, Edson \\ Borges $\mathrm{Jr}^{1,2}$ \\ ${ }^{1}$ Fertility - Centro de Fertilização Assistida, São Paulo/SP, Brazil \\ ${ }^{2}$ Instituto Sapientiae - Centro de Estudos e Pesquisa em Reprodução Assistida, São Paulo/SP, Brazil \\ ${ }^{3}$ Faculdade de Ciências Médicas da Santa Casa de São Paulo, São Paulo/SP, Brazil
}

\begin{abstract}
Objective: To evaluate if ICSI outcome are influenced by the number of cycles and oocyte injected per day.

Methods: Data of ICSI cycles performed in an unselected infertile population were included. The influences of the number of cycles and number of injected oocytes on the ICSI outcomes (fertilization rate, high quality embryos rates on day 3 of development, blastocyst formation rate, the proportion of cycles with embryo transfer, pregnancy rate and implantation rate) were investigated.

Results: Regression analyses results showed no significant influence of the number of cycles and oocytes injected per day on the ICSI outcomes.

Conclusion: The outcomes of ICSI are not influenced by the number of cycles or the number of oocytes injected per day. We suggest that an appropriate number of embryologists per cases per year guarantee successful outcomes independently of the daily workload.
\end{abstract}

Keywords: Fertilization, ICSI, oocyte, pregnancy, workload.

\section{INTRODUCTION}

The intracytoplasmic injection (ICSI) has become a well-established method of in vitro fertilization (IVF) and, over the past decades, the treatment of choice for many infertile couples.

The success of the main important step of ICSI, the spermatozoon injection per se, occurs inside the IVF laboratory and relies on the embryologist's hands. In fact, one of the most significant challenges in assisted reproduction technology (ART) is achieving technical proficiency in micromanipulation (Braga et al., 2007). In addition, the stipulation of a minimum number of employees needed for the laboratory department is necessary. The staff must be adjusted according to the number of cycles performed and the nature of procedures performed in the laboratory (Keck et al., 2005).

Despite the fact that ovarian stimulation is controlled, it is not always predictable. Because the day of hCG injection depends mainly on the patient's ovarian response to gonadotropins, the day of oocyte retrieval cannot be determined in advance. Therefore many cycles are scheduled for the same day, resulting in a high number of oocytes to be injected. Whether the number of ICSI cycles and or the number of injected oocytes per day may be a cause of stress and tiredness for the embryologists, leading to the impairment of ICSI outcome, is still to be elucidated. The aim of this study was to evaluate if ICSI outcome are influenced by the number of cycles and oocyte injected per day.

\section{MATERIAL AND METHODS} teria

Experimental design, patients and inclusion cri-

Data of ICSI cycles performed from January 2010 to
December 2013 in an unselected infertile population were included in this retrospective cohort study. The influences of the number of cycles and number of injected oocytes on the ICSI outcomes (fertilization rate, high quality embryos rates on day 3 of development, blastocyst formation rate, the proportion of cycles with embryo transfer, pregnancy rate and implantation rate) were investigated.

Written informed consent was obtained, in which patients agreed to share their outcomes for research purposes, and the study was approved by the local institutional review board.

\section{Controlled ovarian stimulation}

Ovarian stimulation was achieved by the administration of a recombinant follicle-stimulating hormone (r-FSH, Gonal- $\mathrm{F}^{\circledR}$, Serono, Geneve, Switzerland) on a daily basis until the visualization of at least one follicle $\geq 14 \mathrm{~mm}$, at which time, we began the administration of a gonadotropin-releasing hormone (GnRH) antagonist, cetrorelix acetate (Cetrotide; Serono Laboratories, Geneva, Switzerland).

The ovulation trigger was given by the injection of recombinant human chorionic gonadotrophin (hCG, Ovidrel $^{\mathrm{TM}}$, Serono, Geneve, Switzerland) when at least three follicles $\geq 17 \mathrm{~mm}$ were observed. Oocyte retrieval was performed 35 hours after the administration of hCG, through transvaginal ultrasonography.

\section{Semen sample collection and preparation}

Semen samples were collected in the laboratory and were evaluated for sperm concentration, motility and morphology. Sperm samples were prepared using a 2-layered density gradient centrifugation technique (50\% and 90\%; Isolate, Irvine Scientific, Santa Ana, CA, USA).

\section{ICSI}

ICSI was performed according to Palermo et al (1992). Sperm selection in was analysed under 400x using an inverted Nikon Eclipse TE 300 microscope. The injection was performed in a micro-injection dish prepared with $4 \mu \mathrm{L}$ droplets of buffered medium (Global ${ }^{\circledR}$ w/HEPES, LifeGlobal, Connecticut, USA) and covered with paraffin oil on a heated stage at $37.0 \pm 0.5^{\circ} \mathrm{C}$ in an inverted microscope.

Fertilisation, embryo quality and embryo transfer

Fertilisation was confirmed by the presence of two pronuclei (PN) and the extrusion of the second polar body approximately 16 hours after ICSI.

High-quality embryos were defined as those possessing 4 blastomeres and 8-10 blastomeres on days 2 and 3 of development, respectively, less than $15 \%$ fragmentation, and symmetric and mononucleated blastomeres.

Embryos were placed in a $50-\mu \mathrm{L}$ drop of culture medium (Global ${ }^{\circledR}$, LifeGlobal, CT, USA) supplemented with $10 \%$ protein supplement and covered with paraffin oil in a humidified atmosphere under $7.5 \% \mathrm{CO}_{2}$ at $37^{\circ} \mathrm{C}$ for five days. 


\begin{tabular}{|c|c|c|c|c|}
\hline Variable & Mean & SD & Min & Max \\
\hline ICSI cycles per day & 3.3 & 1.6 & 1 & 10 \\
\hline Injected oocytes per day & 24.4 & 15.1 & 1 & 73 \\
\hline Female age (years) & 36.5 & 5.2 & 20 & 57 \\
\hline Male age (years) & 38.7 & 6.6 & 19 & 71 \\
\hline Female BMI & 24.3 & 3.9 & 16.6 & 43.3 \\
\hline Follicles & 13.8 & 12.3 & 0 & 89 \\
\hline Retrieved oocytes & 9.7 & 9.1 & 0 & 56 \\
\hline Injected oocytes per patient & 7.5 & 5.4 & 0 & 40 \\
\hline Mature oocytes rate (\%) & 74.4 & 22.1 & 0 & 100 \\
\hline Fertilization rate $(\%)$ & 71.6 & 26.1 & 0 & 100 \\
\hline High-quality embryos on D3 (\%) & 38.7 & 29.1 & 0 & 100 \\
\hline Blastocyst formation rate (\%) & 47.4 & 26.5 & 0 & 100 \\
\hline Transferred embryos & 1.9 & 1.1 & 0 & 4 \\
\hline Implantation rate & 18.5 & 30.9 & 0 & 100 \\
\hline \multicolumn{5}{|c|}{$\mathbf{n} / \mathbf{N}$} \\
\hline Cycles with embryo transfer (\%) & \multicolumn{4}{|c|}{$2364 / 2835(83.4)$} \\
\hline Pregnancy rate $(\%)$ & \multicolumn{4}{|c|}{$776 / 2364(32.8)$} \\
\hline Miscarriage rate $(\%)$ & \multicolumn{4}{|c|}{ 92/776 (11.9) } \\
\hline
\end{tabular}

Note: SD: standard deviation; BMI: body mass index.

Embryo transfer was performed on day 5 of development using a soft catheter with transabdominal ultrasound guidance. One to four embryos were transferred per patient, depending on embryo quality and maternal age.

\section{Clinical follow-up}

A pregnancy test was performed 10 days after embryo transfer. All women with a positive test received a transvaginal ultrasound scan after 2 weeks. A clinical pregnancy was diagnosed when the foetal heartbeat was detected. Implantation rates were calculated per patient. Pregnancy rates were calculated per transfer. Miscarriage was defined as pregnancy loss before 20 weeks.

\section{Data analysis and statistics}

Data are expressed as the mean \pm standard deviation for continuous variables, while percentages are used for categorical variables.

Linear regression analysis was used to investigate the influence of the number of cycles and injected oocytes on the continuous variables, such as the fertilization rate, high-quality embryos rate on D3, blastocyst formation rate and implantation rate. The results are expressed as regression coefficients (RC), $r^{2}(\%)$ and $P$ values.

Binary regression analysis was used to investigate the influence of the number of cycles and injected oocytes on the dichotomous variables, such as the proportion of cycles with embryo transfer and the pregnancy rate. The results are expressed as odds ratio (OR), with $95 \%$ confidence interval $(\mathrm{CI})$ and $P$ values.

A $P<0.05$ was considered to be statistically significant. Data analyses were carried out using the Minitab ${ }^{\circledR}$ version 16 statistical program.

\section{RESULTS}

Data of 2835 ICSI cycles, performed between January 2010 and December 2013, in an unselected infertile population, were included in this study. The mean number of cycles performed per day was $3.3 \pm 1.6$ and the mean number of oocytes injected per day was $24.4 \pm 15.1$. Demographic characteristics and ICSI outcomes are shown in Table 1.

Regression analyses results showed no significant influence of the number of cycles and oocytes injected per day on the ICSI outcomes (Table 2 and 3, respectively).

\section{DISCUSSION}

In this study we investigated whether the number of ICSI cycles and or the number of injected oocytes per day may impair ICSI outcome. No significant influences of the number of cycles and oocytes injected per day on the ICSI outcomes were observed.

Studies related to workload in the embryology laboratory have been previously performed. A study from our group aimed at evaluating whether the outcomes of 327 ICSI cycles were influenced by the day of oocyte retrieval (weekday vs. weekend) (Souza Setti et al., 2012). No significant differences were observed between the groups regarding fertilization rate, implantation rate, pregnancy rate and take-home baby rate. The authors concluded that a well-trained embryologist personnel adhered to staff scheduling ensure a similar outcome independent of the workday on which ICSI is performed.

Similarly to the present study, Exposito et al. (2010) investigated whether the daily activity in the embryology laboratory was related to the outcomes of 1558 ICSI laboratory procedures. The results showed no adverse effects associated with the daily workload.

The ASRM guidelines for human embryology laboratories (Practice Committee of American Society for Reproductive and Practice Committee of Society for Assisted Reproductive, 2008) suggest that a sufficient number of qualified laboratory personnel to perform the work of the laboratory must be employed. Clinics that perform up to 150 retrievals and/or cryopreservation cycles per year there should be a minimum of two qualified embryologists. 


\begin{tabular}{|c|c|c|c|}
\hline Continuous ICSI outcomes & RC & $r^{2}(\%)$ & P-value \\
\hline Fertilization rate & 1.098 & 0.5 & 0.121 \\
\hline High-quality embryos on D3 & -0.265 & 0.0 & 0.902 \\
\hline Blastocyst formation rate & -0.498 & 0.1 & 0.309 \\
\hline Implantation rate & 0.354 & 0.0 & 0.365 \\
\hline Dichotomous ICSI outcomes & OR & CI & P-value \\
\hline Cycles with embryo transfer & 0.99 & $0.93-1.05$ & 0.663 \\
\hline Pregnancy rate & 1.01 & $0.96-1.07$ & 0.581 \\
\hline
\end{tabular}

RC: regression coefficient; OR: odds ratio; CI: confidence interval

\begin{tabular}{|c|c|c|c|}
\hline Continuous ICSI outcomes & RC & $r^{2}(\%)$ & P-value \\
\hline Fertilization rate & 0.123 & 0.5 & 0.133 \\
\hline High-quality embryos on D3 & 0.114 & 0.4 & 0.636 \\
\hline Blastocyst formation rate & -0.064 & 0.2 & 0.231 \\
\hline Implantation rate & 0.062 & 0.1 & 0.138 \\
\hline Dichotomous ICSI outcomes & OR & CI & P-value \\
\hline Cycles with embryo transfer & 1.00 & $1.00-1.01$ & 0.217 \\
\hline Pregnancy rate & 1.00 & $1.00-1.01$ & 0.289 \\
\hline
\end{tabular}

RC: regression coefficient; OR: odds ratio; CI: confidence interval

Three embryologists are required to perform between 151 and 300 cycles per year; four embryologists to perform between 301 and 600 cycles; and from 600 cycles on per year one additional embryologist is needed every 200 additional cycles. Additional laboratory staff may be required if andrological and/or endocrinological duties are assigned (Practice Committee of American Society for Reproductive and Practice Committee of Society for Assisted Reproductive, 2008).

In our clinic we have a total of 6 embryologists (4 in the embryology laboratory and 2 in the andrology laboratory). The 2835 ICSI cycles included in this study were performed within a period of 4 years; therefore a mean number of 709 ICSI cycles were performed per year, which is acceptable according to the recommendation above.

In conclusion, the outcomes of ICSI are not influenced by the number of cycles or the number of oocytes injected per day. We suggest that an appropriate number of embryologists per cases per year guarantee successful outcomes independently of the daily workload.

\section{CONFLICT OF INTERESTS}

The authors declare that they have no conflict of interest.

\section{Corresponding author:}

Edson Borges Jr.

Fertility - Assisted Fertilization Center

Av. Brigadeiro Luis Antonio, 4545

São Paulo/SP, Brazil

E-mail: edson@fertility.com.br

\section{REFERENCES}

Braga DP, Pasqualotto FF, Madaschi C, Bonetti TC, Rodrigues $D$, Iaconelli $A$, Jr., Borges $E$, Jr. Use of pig oocytes for training new professionals in human assisted reproduction laboratories. Fertil Steril. 2007;88: 1408-12.

Exposito A, Matorras R, Mendoza R, Crisol L, Martinez-Astorquiza T, Prieto B. Daily workload in the embryology laboratory and in vitro fertilization results. J Reprod Med. 2010;55:49-54.

Keck C, Fischer R, Baukloh V, Alper M. Staff management in the in vitro fertilization laboratory. Fertil Steril. $2005 ; 84: 1786-8$.

Palermo G, Joris H, Devroey P, Van Steirteghem AC. Pregnancies after intracytoplasmic injection of single spermatozoon into an oocyte. Lancet. 1992;340:17-8.

Practice Committee of American Society for Reproductive M, Practice Committee of Society for Assisted Reproductive T. Revised guidelines for human embryology and andrology laboratories. Fertil Steril. 2008;90:S45-59.

Souza Setti A, Paes de Almeida Ferreira Braga D, de Cassia Savio Figueira R, Iaconelli A, Jr., Borges E, Jr. Do weekend oocyte retrievals have an impact on the intracytoplasmic sperm injection cycles outcome? Arch Med Sci. 2012;8:368-70. 